Kirsti Krügener*, Jan Ornik, Roksana Jachim, Bettina Kietz, Karin Petersen, Daniel M. Mittleman, Martin Koch and Wolfgang Viöl

\title{
Monitoring fungus infestation of common beech wood using terahertz radiation
}

https://doi.org/10.1515/hf-2019-0204

Received August 10, 2019; accepted October 18, 2019

\begin{abstract}
Wood is one of the most widely used materials for construction projects, in the furniture industry, as well as in the field of cultural property of valuable objects. Terahertz $(\mathrm{THz})$ radiation has great potential for non-contact and non-destructive analysis of wooden materials. This article reports a study of the degree of growth of Trametes versicolor in wood samples, using $\mathrm{THz}$ radiation. The analysis compares the $\mathrm{THz}$ results to microscopic examinations of the samples, common beech incubated with T. versicolor, at different growth times. The results indicate a strong correlation between the parameters extracted from $\mathrm{THz}$ measurements and the progress of the fungal infestation, suggesting that $\mathrm{THz}$ imaging may be a valuable tool for non-contact inspection of wood.
\end{abstract}

Keywords: common beech, Trametes versicolor, terahertz radiation, wood

\section{Introduction}

The woodworking industry has an increasing interest in processing high-grade damage-free woods of the highest

\footnotetext{
*Corresponding author: Kirsti Krügener, Faculty of Natural Sciences and Technology, HAWK University of Applied Sciences and Arts, Von-Ossietzky-Str. 99, 37085 Göttingen, Germany,

e-mail: kirsti.kruegener@hawk.de

Jan Ornik and Martin Koch: Department of Physics and Material

Sciences Center, Philipps-Universität Marburg, Renthof 5, 35032

Marburg, Germany

Roksana Jachim and Wolfgang Viöl: Faculty of Natural Sciences and Technology, HAWK University of Applied Sciences and Arts, Von-Ossietzky-Str. 99, 37085 Göttingen, Germany Bettina Kietz: Faculty of Resource Management, HAWK University of Applied Sciences and Arts, Büsgenweg 1a, 37077 Göttingen, Germany

Karin Petersen: Faculty of Architecture, Engineering and Conservation, HAWK University of Applied Sciences and Arts, Bismarckplatz 10/11, 31135 Hildesheim, Germany

Daniel M. Mittleman: School of Engineering, Brown University, 184 Hope St., Providence, RI 02912, USA
}

quality. In particular, woods that are used for furniture production are in the upper price segment. Production of veneer and molded wood are examples of wood products used for high-quality furniture production. One challenge involves the structural weakening of the wood products which often takes place some period of time after the product is manufactured. This weakening appears as cracks due to the degradation of lignin and carbohydrates, often as a consequence of infestation by wood fungi (Lohmann 2010). Therefore, when selecting wood, careful attention must be paid to the structural strength and integrity, as well as to the possibility of internal fungal infestation. The development of new methods for non-destructive evaluation of wood will be valuable for such considerations.

This paper presents the results of a study to monitor fungus infestation of wood using terahertz $(\mathrm{THz})$ timedomain spectroscopy (TDS). The THz range of the electromagnetic spectrum (corresponding roughly to $0.3-10 \mathrm{THz}$ ) lies between the infrared and microwave regions (Mittleman 2017). This spectral range has recently been exploited for a variety of applications involving the non-destructive evaluation of dry, non-metallic materials (Duling and Zimdars 2009). The radiation is readily able to penetrate many non-ionic and non-conducting materials. In imaging applications, the spatial resolution is typically sub-millimeter in scale (Mittleman 2018). Moreover, THz radiation is non-ionizing and (at low power) poses no threat to living tissue, and is therefore suitable for operation without cumbersome radiation monitoring tools or concerns about health or safety. Given the value and uniqueness of some materials such as art pieces, it is important to develop new non-invasive diagnostic methods (Krügener et al. 2017). In addition to the investigation of samples using $\mathrm{THz}$ spectroscopy, the mass change of samples due to wood decomposition was recorded, and optical observation of decomposition on thin sample sections with a light microscope was performed.

This study focuses on common beech (Fagus sylvatica L.) as a test wood type, as it is one of the most common construction timbers. It is also a widespread type of tree. For instance, about $15 \%$ of the total forest area in Germany comprises beech trees (Forest Report of 
the Federal Government 2017). The test fungus species used here is the white-rot fungus Trametes versicolor, also called butterfly tramete, as it is distributed worldwide in all climates and is found on many hardwoods, especially beech. It is a common test fungus according to European standard ÖNORM EN 113 for hardwood samples (Schmidt 2006). It invades living trees through wounds and after branch cut. Therefore, it is also called a wound parasite. Trametes versicolor degrades lignin, cellulose and hemicelluloses almost simultaneously and is therefore known as simultaneous white-rot fungus. In common beech wood, the fungal hyphae grow primarily in vessels along the rays of the wood, starting in the earlywood. The latewood degradation is delayed with respect to the earlywood degradation, but follows the same degradation pattern. Trametes versicolor triggers a so-called white rot with black demarcation lines (Schwarze and Arnold 2011). White-rot fungi are the only organisms that can split the aromatic rings and introduce the opened rings into their metabolism which gives wood its strength and stability. Wood with this type of degradation cannot be used for high-end products.

\section{Materials and methods}

Samples of beech wood were acquired from Göttinger Forest/ Leinebergland Lower Saxony (Germany). These were sawed into slices of approximately $30 \mathrm{~cm}$ diameter, and then transported to Hildesheim for further processing. After 6 weeks in normal storage conditions $\left(20^{\circ} \mathrm{C}, 65 \%\right.$ relative humidity), a set of 16 rectangular sample pieces $\left(25 \times 25 \times 5 \mathrm{~mm}^{3}\right)$ were sawed from heartwood. In order to determine the degree of mass loss due to wood decomposition caused by fungus infestation, according to European standard ÖNORM EN 113 , the bone-dry weight of all samples was determined. In order to achieve a suitable wood moisture for the fungus growth, all samples were stored in standard atmosphere $\left(20^{\circ} \mathrm{C}, 65 \%\right.$ relative humidity) for 1 week. Moisture measurement was performed using an electronic wood moisture meter (Voltcraft FM-300 wood moisture meter, Voltcraft, Hirschau, Germany). The samples were vacuum-sealed directly after the standard conditioning in polystyrene/polyamide film and labeled. All specimens were then sterilized in order to exclude the presence of fungus or bacteria and fungi that may interfere with the growth of the test fungus. Sterilization was conducted by using ionizing $\gamma$ radiation at $25 \mathrm{kGy}$ for 5 days.

To grow the test fungus, a nutrient medium was prepared according to German standard DIN EN 113. The nutrient medium was put into Petri dishes, filling them up to $5 \mathrm{~mm}$ in height. They were covered with another Petri dish and sealed with Parafilm ${ }^{\circledR}$ forming a test vessel. All Petri dishes were also sterilized by $\gamma$ radiation. For each wood sample, a separate vessel was prepared. The nutrient medium in test vessels was sterilized in an autoclave at $120^{\circ} \mathrm{C}$ for $20 \mathrm{~min}$ and then stored in the vaccination bank for about 2 days until the nutrient medium solidified. The fungal strain was derived from the culture collection at Hildesheim/Göttingen University Lower Saxony, Germany. The grown fungi cultures were less than 4 weeks old and still growing actively. The grown mycelium was mixed with a sterile micro-turner with $10 \mathrm{ml}$ of $0.9 \%$ sodium hydroxide aqueous solution. Ten microliters of the fungal suspension were uniformly inoculated on the prepared nutrient media within 12 test vessels and stored at $20-21^{\circ} \mathrm{C}$ and $75-85 \%$ relative humidity in mini incubators to avoid cross-contamination. Four test vessels were not inoculated, serving as reference samples.

After 21 days, the inoculated nutrient medium in the test vessels was completely imbued by fungi, which corresponded to the active development phase. A sterilized stainless steel grid was inserted in each test vessel and used as a spacer. A wood sample was placed on the spacer in each test vessel, which was sealed again. The test vessels with samples were kept at $20-21^{\circ} \mathrm{C}$ and $75-85 \%$ relative humidity, letting the fungus grow for 4, 8, 16 and 20 weeks. After this growth time, three wood samples with growing fungus and one reference wood sample were removed from the test vessels. The mycelium adherent on the surface of the wood samples was carefully removed using a scalpel. After the mycelium was removed, the samples were weighed again. The determined mass of a fresh sample corresponded to the mass of both wood and the water inside it. Then, the samples were dried and their bone-dry weight was recorded to determine the degree after fungal culture. Then, $\mathrm{THz}$ transmission measurements were performed, and decomposition was visually inspected under a light microscope.

THz measurements: THz measurements were performed using a time-domain spectrometer SPECTRALYZER ${ }^{\circledR}$ from HÜBNER GmbH \& Co. KG, Kassel, Hesse, Germany. This is a commercial stand-alone system, based on photoconductive antennas and a mode-locked fiber laser (https://www.hubner-photonics.com/products/terahertztechnology/terahertz-spectrometers/t-spectralyzer/, 20019). The operation of this system is typical of TDS systems which have been on the market for nearly two decades (Duling and Zimdars 2009). Such systems produce picosecond pulses of $\mathrm{THz}$ radiation, which can be detected coherently (i.e. the measurement produces a signal proportional to the $\mathrm{THz}$ electric field, not the intensity). As a result, it is possible to obtain both the real and imaginary parts of a sample's (complex) dielectric function $[\tilde{\varepsilon}(v)]$ directly from the measurement, which can be related to the complex refractive index $[\tilde{n}(v)]$ by $\tilde{\varepsilon}(v)=[\tilde{n}(v)]^{2}=[n(v)+\mathrm{i} \kappa(v)]^{2}$, where $n(v)$ is the usual refractive index, $\kappa(v)$ is the extinction coefficient and $v$ is the frequency. The extinction coefficient is directly related to the more familiar absorption coefficient $[\alpha(v)]$ according to $\kappa(v)=\alpha(v) c /(4 \pi v)$, where $c$ is the speed of light in vacuum. Here, the results in terms of the dimensionless values $n(v)$ and $\kappa(v)$ are presented. These functions can be determined over the entire spectral range covered by the spectrometer. For the spectrometer used, this spectral range spans from 0.3 to $4.0 \mathrm{THz}$; however, for the purposes of the measurements discussed here, only a reduced portion of this range, $0.3-0.7 \mathrm{THz}$, is used. This is restricted to the portion where the signal-to-noise is highest. As noted below and reported previously (Krügener et al. 2019), wood samples exhibit no strong frequency-dependent features within this spectral range, so the results are largely independent of these specific spectral limits.

The samples were positioned on a thin foil on a motorized sample holder inside a sample chamber. Automated imaging was performed by moving the samples through the focus point and recording the $\mathrm{THz}$ waveform at each position in a transmission geometry. The real and imaginary parts of the complex refractive index were extracted from the measured data, accounting for both the Fresnel coefficients and the propagation through the sample. Assuming that the real part 
of the refractive index $n$ is much greater than the imaginary part $\kappa$, the two parts of the refractive index can be analytically calculated (Naftaly and Miles 2007). Due to the fact that the samples were relatively thick (around $5 \mathrm{~mm}$ ) and consequently strongly attenuated the signal at higher frequencies, the refractive index was extracted only in the frequency range from 0.3 to $0.7 \mathrm{THz}$, where the spectrometer had the highest dynamic range (as noted earlier). For the calculation of the refractive index, the sample thickness was determined using a caliper with a resolution of $0.02 \mathrm{~mm}$.

As measurements were performed with a focused $\mathrm{THz}$ beam with an approximate spot size of $1.5 \mathrm{~mm}$, approximately 64 measurements per sample were acquired. From these measurements, an average value and standard deviation were computed for each of the extracted dielectric values $n$ and $\kappa$, for each sample. As standard deviation is calculated based on the evaluation of many points across the sample, it takes sample's inhomogeneity into account. Furthermore, wood is an anisotropic material resulting in birefringence of up to 0.1 with the fast and slow axes perpendicular and parallel to the sample's grain, respectively (Todoruk et al. 2008). As the sample's grain was not straight and changed orientation across the sample, the standard deviation takes also polarization dependence of the results into account.

Mass measurements: It was expected that the mass of the samples would change with the fungus growth. One reason for the change is the wood degradation and a second is change in water content in the wood. In order to distinguish these two reasons, the bone-dry mass of each sample was determined before the infestation. After the infestation and removal of the adherent mycelium, the wet mass as well as the bone-dry mass of the samples were measured. Comparing the bone-dry mass before $\left(m_{\mathrm{dry}, 1}\right)$ and after $\left(m_{\mathrm{dry}, 2}\right)$ the infestation, the mass loss due to the infestation can be calculated as:

$$
\text { Mass loss }=\frac{m_{\mathrm{dry}, 1}-m_{\mathrm{dry}, 2}}{m_{\mathrm{dry}, 1}}
$$

Visual inspection of the samples after the degradation test: In order to be able to observe the mycelium growth and degradation of wood, thin sections were prepared and evaluated under the transmission light microscope. The basics of the evaluations were made based on Bavendamm (1936) and Rypácek (1966). The definition of the degradation pattern was performed in the chronological course of the fourth, eighth, $16^{\text {th }}$ and $20^{\text {th }}$ incubation week. All analyses were performed including the reference samples.

Production of thin sections: The samples were cut using a sled microtome with interchangeable blades. The cutting thickness was about $20 \mu \mathrm{m}$ with an inclination angle of the knife of about $10^{\circ}$ and a cutting angle of $40-50^{\circ}$. Both radial and tangential sections have been studied. In order to stabilize the wood prior to investigation, the samples were inserted into polyethylene glycol $\mathrm{C}_{2} \mathrm{H}_{4} \mathrm{O}$. The sections themselves were made on dry samples. The cut sections were placed on the slide in a mixture of saturated aqueous aniline blue and lactic acid (mixing ratio 2:1) pipetted directly onto the glass, and briefly heated twice by means of a Bunsen burner. The sections were then rinsed with $\mathrm{H}_{2} \mathrm{O}$.

The blue color accentuates the wood structures and the fungus hyphae stand out more. Subsequently, the stained sections were dehydrated $2 \times 3 \mathrm{~min}$ and $1 \times 1 \mathrm{~min}$ in isopropanol $\mathrm{C}_{3} \mathrm{H}_{8} \mathrm{O}$ and embedded with Euparal to produce the sections as permanent preparations.
To allow the resin to cure, the slides with the cuts were dried at $60^{\circ} \mathrm{C}$ for 1 week.

\section{Results and discussion}

For all samples, the measured refractive index $n(v)$ is essentially independent of frequency within the relevant frequency range $(0.3-0.7 \mathrm{THz})$. Therefore, it is reasonable to average the value over this entire frequency range, reducing the data to a single averaged value. In addition, the measured extinction coefficients $\kappa(v)$ exhibit a very weak (nearly linear) increasing trend with frequency. This trend is weak enough that it is reasonable to perform a similar spectral averaging. Using these averages, both the refractive index and the extinction coefficient exhibited a consistent trend with respect to the time of fungus infestation: the values of both $n$ and $\kappa$ decrease with increasing infestation time, with a few exceptions. The average refractive index of non-infested dry wood samples was determined to be $1.42 \pm 0.03$. This value decreased to $1.09 \pm 0.01$ after 20 weeks of infestation. The average extinction coefficient changed from $0.055 \pm 0.0065$ for non-infested samples to $0.018 \pm 0.0024$ after 20 weeks of infestation. These extinction coefficient values are much less than unity, which justifies the approximation that $n<<\kappa$, as noted earlier. A similar trend is also observed for the mass of most of the samples. Figure 1 shows the mass loss (in percentage) vs. infestation time.

The observed trend of mass loss is not surprising, as fungi cause decomposition of wood as their source of energy, resulting in mass and density reduction (i.e. increased mass loss). The speed at which fungi grow can

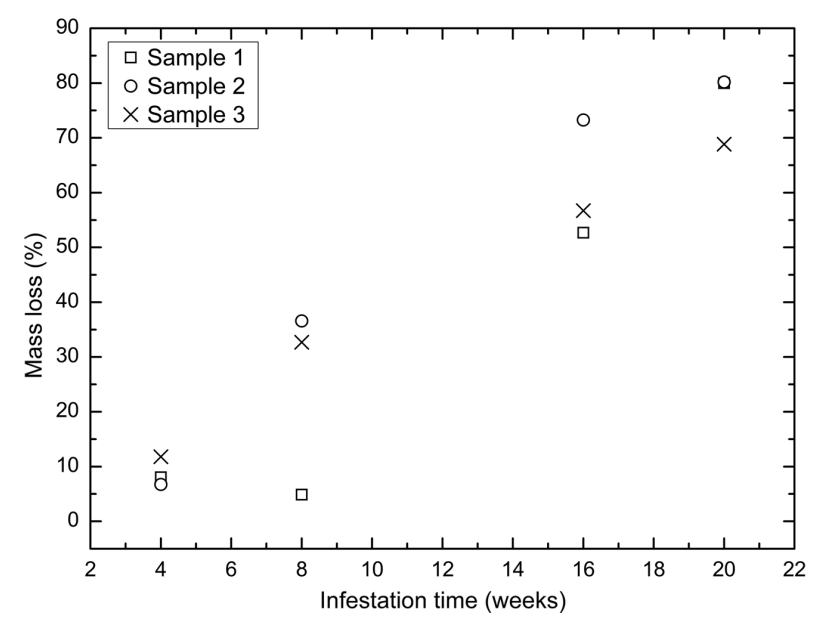

Figure 1: Mass loss of the samples at different infestation times. For reference samples (non-infested), the mass loss equaled zero within $2 \%$ tolerance (not shown in the plot). 
vary, despite taking care that all samples were prepared in the same way. This fact contributes to the scatter in the data.

In many previous studies, it is empirically observed that both the $\mathrm{THz}$ refractive index and extinction coefficient are related to the density of the material (Koch et al. 1998; Naftaly and Miles 2007; Palermo et al. 2008; Laib and Mittleman 2010; May et al. 2013). Thus, it is logical to seek a correlation between the measurements of refractive index and extinction coefficient and the measured mass loss. First, the refractive index loss and extinction coefficient loss are computed as:

$$
\begin{aligned}
& \text { Relative refractive index loss }=\frac{n_{1}-n_{2}}{n_{1}-1} \\
& \text { Extinction coefficient loss }=\frac{\kappa_{1}-\kappa_{2}}{\kappa_{1}},
\end{aligned}
$$

where $n_{1}$ and $\kappa_{1}$ are the spatially and spectrally averaged refractive index and extinction coefficient of noninfested wood samples, respectively, and $n_{2}$ and $\kappa_{2}$ are the averaged refractive index and extinction coefficient of an infested sample, respectively. Note that a value of $n_{\text {vacuum }}=1$ is subtracted from $n_{1}$ in the denominator of equation (2). If the entire sample was completely degraded by the fungal infestation, the refractive index $n_{2}$ would be 1 (equivalent to no sample), which would correspond to $100 \%$ mass loss. Figure 2 shows the results, displaying the correlation between refractive index loss and mass loss (Figure 2a) and the correlation between extinction coefficient loss and mass loss (Figure 2b). In both cases, linear function fitted the data well with an $\mathrm{R}^{2}$ value of 0.99 . Evidently, the results exhibit a very strong correlation in both cases. In addition, a Monte Carlo approach was employed to determine the correlation coefficient between optical parameter loss and mass loss taking also the measurement uncertainties into account. In short, many random datasets normally distributed within the error bars are created and an average correlation coefficient and its standard deviation are calculated. We found a correlation coefficient of $0.88 \pm 0.05$ for the refractive index and $0.69 \pm 0.14$ for the extinction coefficient. These results suggest that $\mathrm{THz}$ spectroscopy could be a valuable non-contact and nondestructive tool for the assessment of mass loss due to fungal infestation of wood.

Evaluation of the microscopic analysis: In the common beech wood, three types of degeneration by T. versicolor were observed:

1. round to oval cavities,

2. fine growth through cell walls,

3. planar cell wall degradation starting from the lumen, which expands with time.

Observing this biotic degradation confirms that the fungus infestation took place in a foreseen way (Tuor et al. 1995). The results are shown in Figures 3-5. Hyphae growth took place before the degradation of wood components, which began in the vessels and proceeded from there into the parenchyma and fiber cells. The mycelium density was already high by the 4-week mark, increased
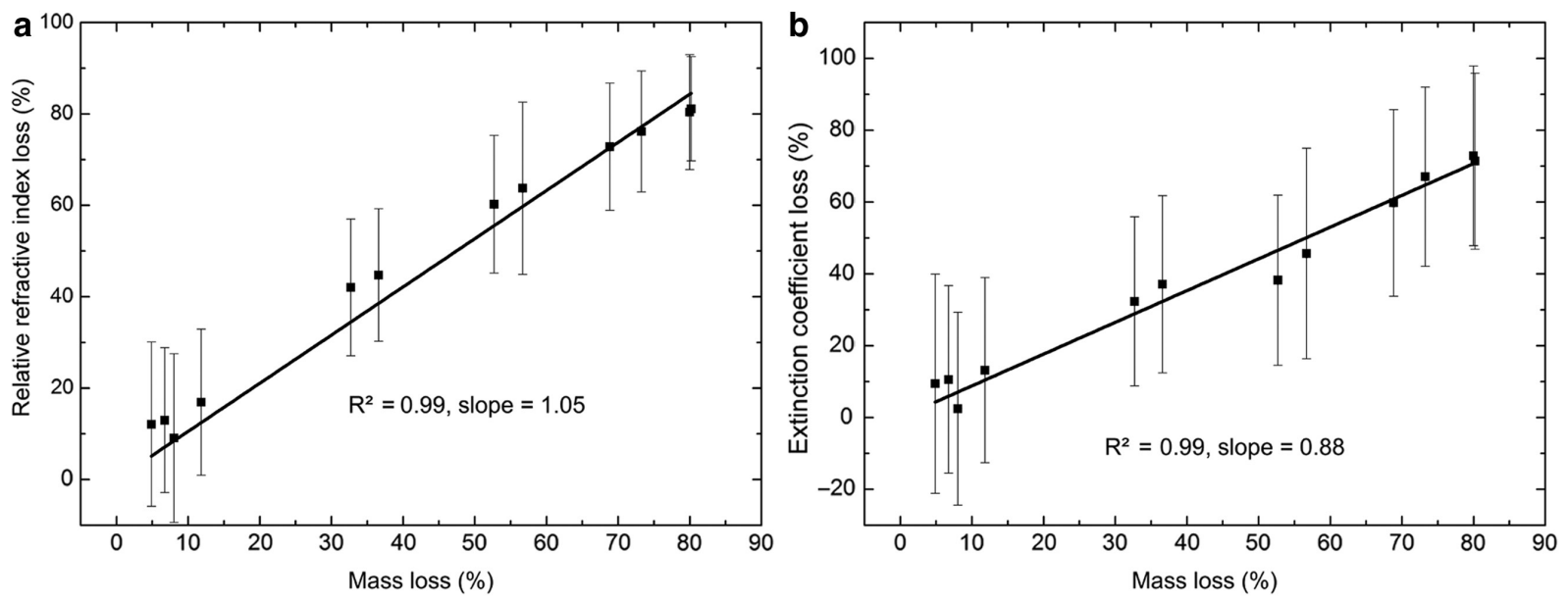

Figure 2: Correlation of dielectric parameter loss with mass loss of the samples.

(a) Relative refractive index loss vs. mass loss with a linear fit, (b) extinction coefficient loss vs. mass loss with a linear fit. In both cases the linear function with a fixed intercept at $(0,0)$ was fitted and the coefficient of the determination $\left(R^{2}\right)$ was 0.99 . Slope was determined as 1.05 and 0.88 in the case of relative refractive index loss and extinction coefficient loss, respectively. 


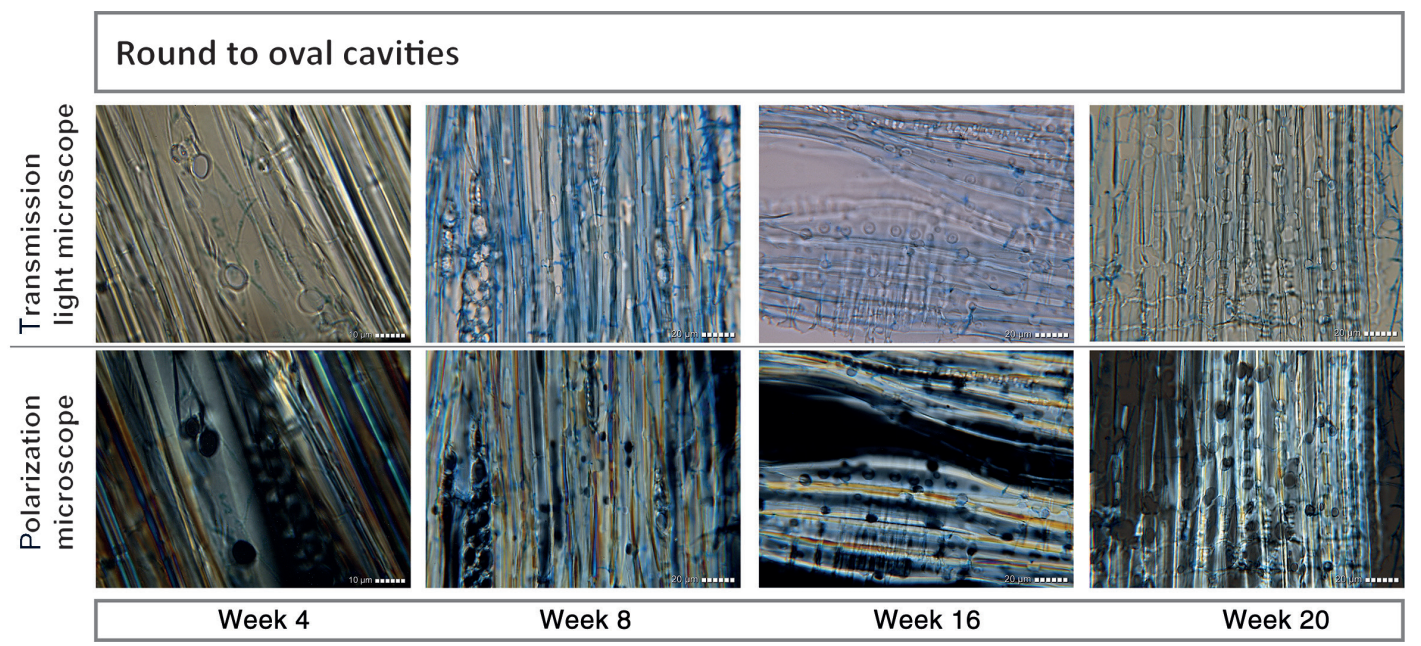

Figure 3: The number of round cavities increases significantly over time.

After 4 weeks they are sparse, moderately frequent after 8 weeks, more frequent after 16 weeks and numerous after 20 weeks.

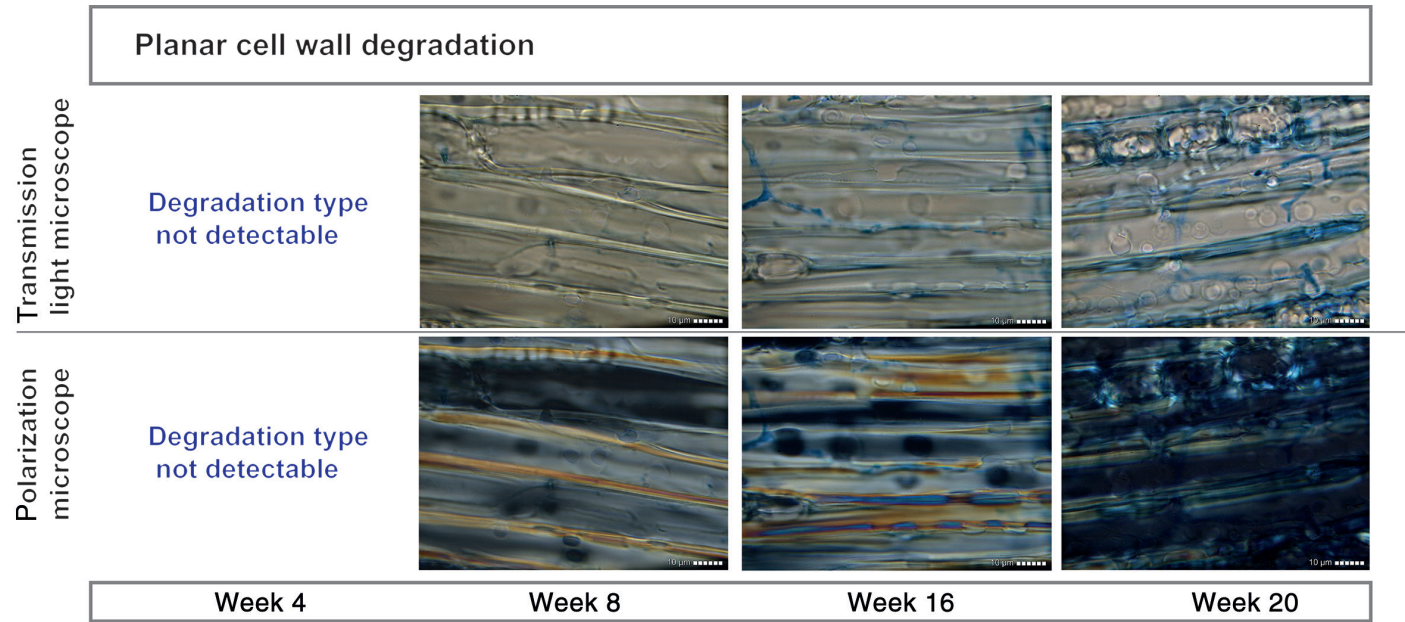

Figure 4: In the fourth week, few caverns and many fine wall penetrations were visible.

These can merge into each other over time.

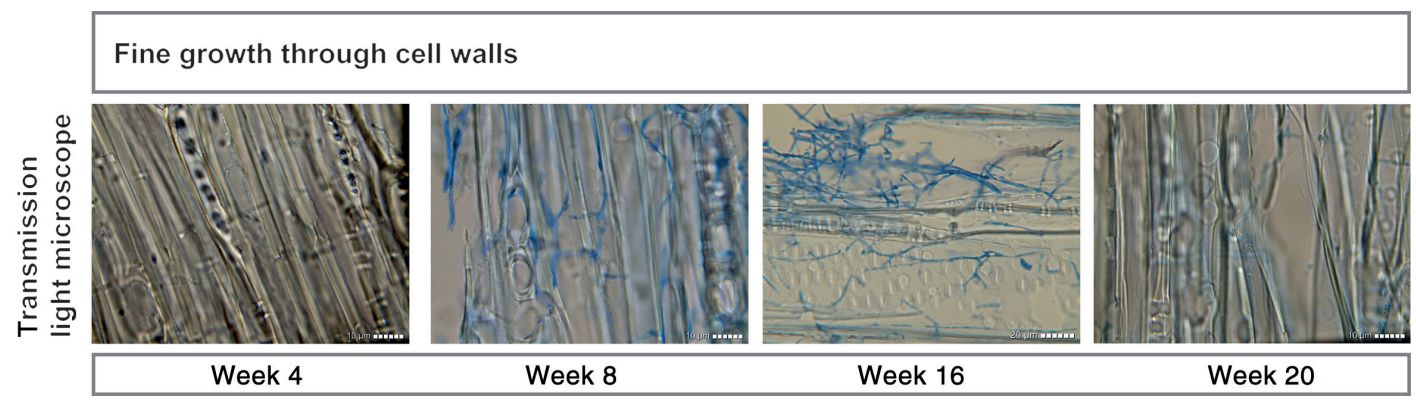

Figure 5: The first indications of a planar cell wall degradation appear after 8 weeks and become clear after 16 weeks. After 20 weeks, it was the most dominant feature of the damage picture.

until the eighth week, stagnated in the $16^{\text {th }}$ week and decreased or remained at the same level at the $20^{\text {th }}$ week. The hyphal diameter increased to $2-4 \mu \mathrm{m}$ until the eighth week and then stabilized, with the mycelete density in the parenchymal cells and fibers being low and then variable. The colonization was very dynamic and was then partially 
decomposed autolytically (dissolving of hyphae). When looking at the cutting directions, there were no substantial differences in the degradation patterns. However, the trend shown in the $\mathrm{THz}$ analyses and mass loss measurements can be confirmed in the microscopic analyses. In the following, the changes in the individual degradation patterns of the weeks are compared in the microscopic analysis.
In this comparison, the results for the control samples are not presented.

In the comparison of non-infested beech wood of a control sample and infested material of a sample both from the $20^{\text {th }}$ week, it becomes clear how strongly the degradation of the cell walls took place (Figure 6).

The microscopic analysis was performed on a single infested sample from each test group (i.e. infestation

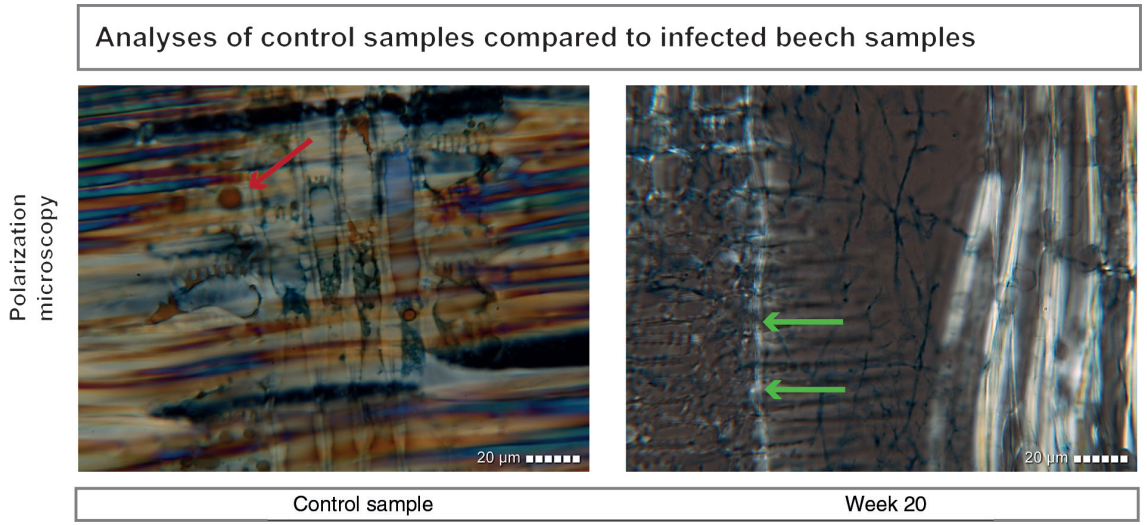

Figure 6: Left side, Control sample junction field and fibrous tissue with brown deposits (red arrow). Right side, Infected wood sample ( 20 weeks) cell wall rejuvenation by the flat cell wall degradation (green arrows) and round to oval cavities of white-rot fungus. Visible are numerous hyphae.

Table 1: Results of visual inspection.

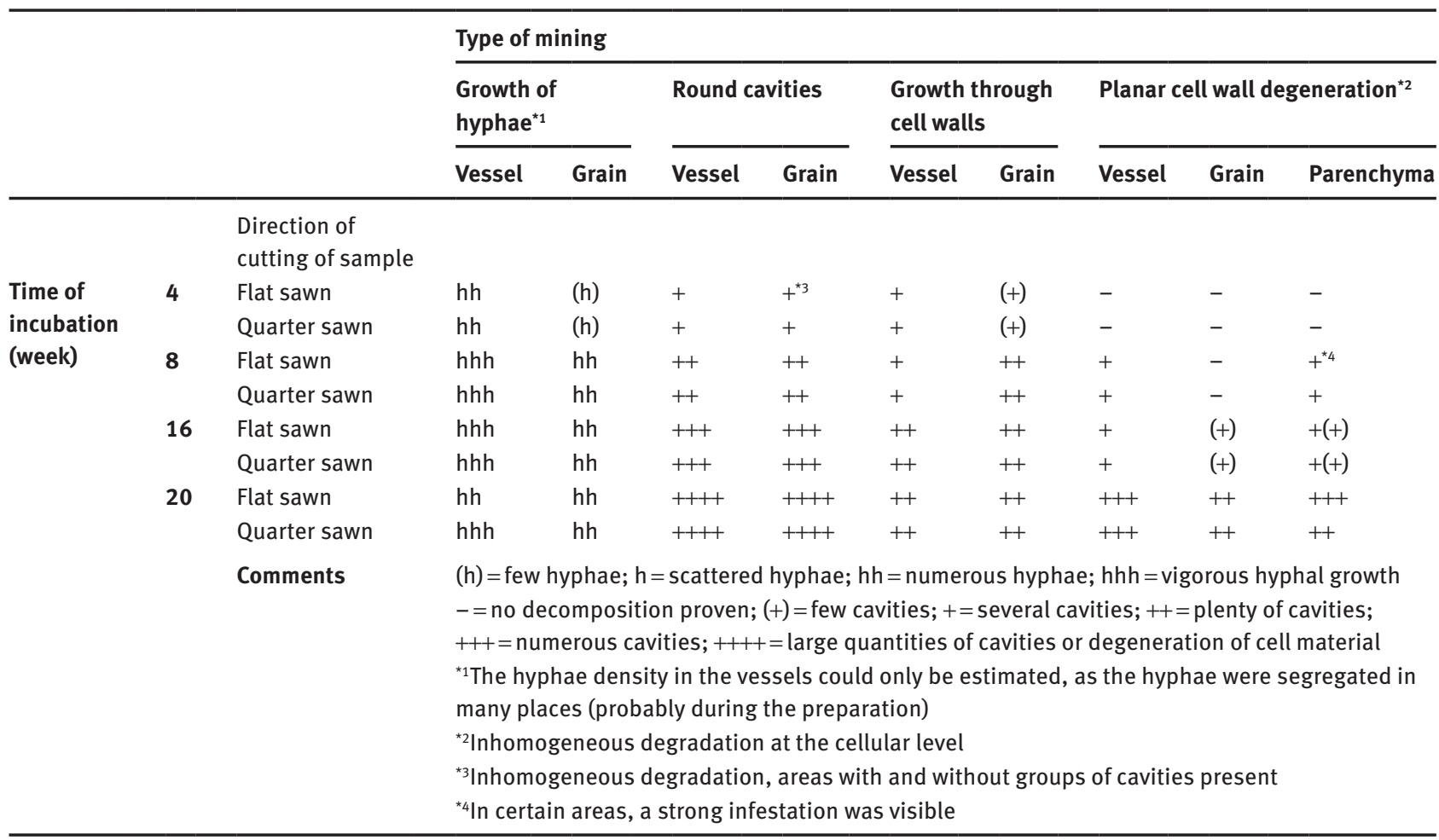

Patterns of cellular degradation with assignment of the damaged cell structures. Fungus: Trametes versicolor; wood species: Fagus sylvatica. 
time of $4,8,16,20$ weeks) and a single reference (noninfested) sample. This means that in total five samples were investigated under a microscope, as the sample preparation for the microscopic investigation as well as investigation on its own is quite time-consuming. The selected samples followed the trend of losing mass and reducing refractive index with increasing infestation time, which was also qualitatively confirmed with the microscopic analysis. The evaluation of the results of the microscopic analysis are summarized in Table 1.

\section{Conclusion}

These results show that the effect of fungal growth on the wood structure in the early stage of growth can be studied using $\mathrm{THz}$ radiation. Based on the THz data, it is possible to distinguish between infested and non-infested wood. Especially with regard to the reduced stability of wood without surface mycelium, the non-destructive and non-contact evaluation using $\mathrm{THz}$ radiation can be an important tool for the quality verification of certain wood materials. Thus, the THz technique presented here could help to investigate, for example, art or historical objects, or even thin wooden materials used for the construction of furniture. In both cases, inspection should be non-destructive because the materials are too valuable in the case of art and cultural heritage and too costly in the case of industrial production. For both applications, the use of mobile THz systems already on the market would be a feasible solution. It is worth mentioning that $\mathrm{THz}$ investigation is much faster than the destructive microscopic analysis. It is possible that other species of fungi and wood can also be investigated using this method. Finally, although a broadband $\mathrm{THz}$ source was used for these studies, it is worth noting that a less expensive narrowband source may produce similar results, and may therefore be more convenient in some situations.

Acknowledgments: The authors would like to thank Professor Rolf Kehr for providing fungus cultures.

Author contributions: All the authors have accepted responsibility for the entire content of this submitted manuscript and approved submission.

Research funding: The authors would like to thank the Ministry for Science and Culture of Lower Saxony and the Funding of VW “Niedersächsisches Vorab” (Germany) for their financial support. Also, DMM gratefully acknowledges the support from the Alexander von Humboldt Foundation.

Employment or leadership: None declared.

Honorarium: None declared.

\section{References}

Bavendamm, W. Erkennen, Nachweis und Kultur der holzverfärbenden und holzzersetzenden Pilze. Urban \&Schwarzenberg, Berlin, 1936.

Duling, I., Zimdars, D. (2009) Terahertz imaging: revealing hidden defects. Nat. Photonics 3:630.

Forest Report of the Federal Government. Forest Report of the Federal Government 2017. Berlin, 2017.

Koch, M., Hunsche, S., Schumacher, P., Nuss, M.C., Feldmann, J., Fromm, J. (1998) THz-imaging: a new method for density mapping of wood. Wood Sci. Technol. 32:421-427.

Krügener, K., Busch, S.F., Soltani, A., Castro-Camus, E., Koch, M., Viöl, W. (2017) Non-destructive analysis of material detachments from polychromatically glazed terracotta artwork. J. Infrared Millimeter Terahertz Waves 38:495.

Krügener, K., Sommer, S., Stübling, E., Jachim, R., Koch, M., Viöl, W. (2019) THz properties of typical woods important for European forestry. J. Infrared Millimeter Terahertz Waves 40:770.

Laib, J.P., Mittleman, D.M. (2010) Temperature-dependent terahertz spectroscopy of liquid n-alkanes. J. Infrared Millimeter Terahertz Waves 31:1015-1021.

Lohmann, U. Holz-Lexikon. Nikol, Hamburg, 2010.

May, R.K., Su, K.E., Han, L., Zhong, S., Elliott, J.A., Gladden, L., Zeitler, J.A. (2013) Hardness and density distributions of pharmaceutical tablets measured by terahertz pulsed imaging. J. Pharm. Sci. 102:2179-2186.

Mittleman, D. (2017) Perspective: terahertz science and technology. J. Appl. Phys. 122:230901.

Mittleman, D. (2018) Twenty years of terahertz imaging. Opt. Express 26:9417-9431.

Naftaly, M., Miles, R. (2007) Terahertz time-domain spectroscopy for material characterization. Proc. IEEE 9:1658-1665.

Palermo, R., Cogdill, R.P., Short, S.M., Drennen III, J.K., Taday, P.F. (2008) Density mapping and chemical component calibration development of four-component compacts via terahertz pulsed imaging. J. Pharm. Biomed. Anal. 46:36-44.

Rypácek, V. Biologie Holzzerstörender Pilze. G. Fischer, Jena, 1966.

Schmidt, O. Wood and Tree Fungi. Springer Berlin Heidelberg, Berlin, 2006.

Schwarze, F.W., Arnold, M. (2011) Dauerhaftigkeit von thermisch modifiziertem Holz (TMT).

Todoruk, T.M., Schneider, J., Hartley, I.D., Reid, M. (2008) Birefringence of wood at terahertz frequencies, Proc. SPIE 7099:70992P-12.

Tuor, U., Winterhalter, K., Fiechter, A. (1995) Enzymes of white-rot fungi involved in lignin degradation and ecological determinants for wood decay. J. Biotechnol. 41:1-17. 